\title{
Directed*-topology and Scott*-topology on Transitive Binary Relational Sets
}

\author{
Mohammed Khalaf $\mathbf{M}^{1,2 *}$
}

${ }^{1}$ High Institute of Engineering and Technology King Marioutt P.O. Box 3135, Egypt

${ }^{2}$ Mathematics Department, Faculty of Science in Zulfi, Majmaah University, Zulfi 11932, P.O. Box 1712, Saudi Arabia

\begin{abstract}
In this work we naturally put forth an open question whether one may construct a scott-topology on transitive binary relational sets (so called TRS). We prove that a TRS gives rise to several natural topologies defined in terms of the given TRS structure. Mainly, we consider directed topologies and scott topologies on TRS and their interactions with the continuity property of TRS. Most of our results are generalizations of corresponding results in references as we will illustrate. Sometimes we need pre-ordered sets instead of TRS.
\end{abstract}

Keywords: Poset; Transitive binary relational sets; Directed topologies; Scott topologies; Pre-ordered sets

2010 Mathematics subject classification: 03E72; 18B30; 54A40

\section{Introduction}

In domain and poset [1-3], Scott-topologies were defined. Abramsky and Jung [4] introduced the concepts of continuous directed complete posets (continuous domain) and algebraic domains. Heckmam [1] studied these conceptes by more details and explained a interactions between Scott-topology and these notions. Also, add the concepts of bounded complete posets, bounded complete domains, finitely complete posets, finitely complete domains, finitarily complete posets [5-8]. Hoffmann and Lawson [8-10] gives the concepts of continuous posets. And in more general fashion by Markowsky [11] and Eme [12]. Nino-Salcedo [3], add by deep studies the concept of algebric posets. We note that the concept of continuous posets (resp., algebraic posets) in the sense of Nino-Salcedo and continuous domain (resp., algebraic domain) of $R$ are the same. Zhang [13] studied a type of continuous poset which a generalizations of the continuous poset in the sense of Nino-Salcedo. and add some interactions between bounded complete domains, Scott topology and Lawson topology. This work is devoted to introduce and study the continuity and algebraicness properties of TRS. Our results extended the results in posets and in domains [1-3,13]. The concepts of upper bound (for short $u b$ ), lower bound (for short $l b$ ), least upper bound (for short), gretest lower bound (for short) in any poset are clear also, some concepts in mathematical logics my building some times needs these facts [14].

To solve the problem we first introduce the following concepts.

\section{Definition 1.1}

Let ' $\leq$ ' be a binary relation set on $X \neq \phi$. Then;

(1) ' $\leq$ 'is called reflexive iff $\forall x X, x \leq x$ [14];

(2) ' $\leq$ 'is called antisymetric iff $\forall x, y \in X, x \leq y$ and $y \leq x x=y$ [14];

(3) ' $\leq$ 'is called transitive iff $\forall x, y, z \in X, x \leq y$ and $y \leq z x=z$ [14];

(4) ' $\leq$ 'is called symetric iff $\forall x, y \in X, x \leq y \Rightarrow y \leq x$ [14];

(5) ' $\leq$ 'is called interpolative iff $\forall x, z \in X$, with $x z, \exists y X$ s.t. $x y \leq z[1,15]$.

(6) if ' $\leq$ 'satisfies the conditions (1), (2) and (3), then $(X, \leq)$ is called Partialy order set (Poset) [14];

(7) if' $\leq$ 'satisfies the conditions ( 1$)$, and ( 3$)$, then $(X, \leq)$ is called pre- orderd set (Quasi set) [14];

(8) if ' $\leq$ 'satisfies the conditions (1), (2), (3) and (4), then $(X, \leq)$ is called an equvalence set,

(9) if ' $\leq$ 'satisfies the conditions (3) and (5), then $(X, \leq)$ is a Continuous information system $[15,16]$.

(10) if ' $\leq$ 'satisfies the conditions (3), and $\forall x \in X$, and for every finite subset $A$ of $X$ the following axiom holds: if $\forall y A, y \leq x$ then $\exists z X$ s.t. $\forall y A$, $y \leq z$ and $z x$, then $(X, \leq)$ is abstract basis [17].

\section{Definition 1.2}

Let $A X$. Then:

(1) $A$ is called directed subset of $X$ iff $A \neq \phi$ and $\forall x, y \in A, \exists z A$ s.t. $x z$ and $y z[1]$;

(2) The lower (resp. upper) closure in $X$ of $A$ is denoted by $\downarrow A$ (resp. $\uparrow A$ ) and defined as follows: $\downarrow A=\{x \in X: \exists y A$ s.t. $x \leq y\}$ (resp. $\uparrow A=\{x \in X: \exists y A$ s.t. $y \leq x\})[1]$

(3) The convex hull $A$ is denoted by $\uparrow A$ and defined as follows: $\uparrow A=\downarrow A \uparrow A[1]$;

(4) Let $A, B \subseteq X . B$ is called cofinal in $A$ iff $B A \subseteq \downarrow(B)$ [1].

\section{Definition 1.3}

Let $A X$. Then:

(1) A subset $A$ of the domain [1] (resp. Poset) $X$ is called directed closed ( $d$-closed for short) iff $\forall$ directed subset $D$ of $A,(D) \in A$;

(2) A subset $A$ of the Poset $X$ is called Scott-closed iff $A$ is d-closed lower subset of $X[3]$;

*Corresponding author: Mohammed Khalaf M, Mathematics Department, Faculty of Science in Zulfi, Majmaah University, Zulfi 11932, P.O. Box 1712, Saudi Arabia, Tel: 95140-0582905323, 2293641375; E-mail: Khalfmohammed2003@yahoo.com

Received September 27, 2017; Accepted November 20, 2017; Published November 28, 2017

Citation: Khalaf MM (2017) Directed*-topology and Scott*-topology on Transitive Binary Relational Sets. J Generalized Lie Theory Appl 11: 284. doi: 10.4172/17364337.1000284

Copyright: @ 2017 Khalaf MM. This is an open-access article distributed under the terms of the Creative Commons Attribution License, which permits unrestricted use, distribution, and reproduction in any medium, provided the original author and source are credited. 
(3) A is called d-(resp. Scott-) open iff $A^{c} \mathrm{~d}$-(resp. Scott-) closed [1,3];

(4) Let $x, y \in X$. We say $x$ below (resp. $y$ is way above) $y$ (resp. $x$ ), denoted by $x<<y$ iff $\forall D \subseteq X$, s.t. $D$ is directed subset of $X$ with $D$ exists and $y \leq D, \exists d D$ s.t. $x d$. The family of all elements in $X$ each of which way above (resp. way below) $x$ is denoted and defined as follows: $\Uparrow x=\{y \in X: x<<y\}$ (resp. $\Downarrow x=\{y \in X: y<<x\}[3]$;

(5) Let $x \in X$. If $x<<x$, then $x$ is said to be isolated. The family of all isolated points above (resp. below) $x \in X$ is denoted and defined by: $\uparrow^{\circ} x=\{y \in X: y<<y$ and $x \leq y\}$ resp. $\downarrow 。 x=\{y X: y<<y$ and $y x\}[3]$.

Proposition 1.1: (Proposition 3.5.2 [1]). Let $X$. be a domain, we have:

(1) $X$ and $\phi$ are d-closed;

(2) The intersection of a family of d-closed sets is d-closed; and

(3)The union of two of d-closed sets is d-closed. Then the family of all d-open sets in a domain $X$ is a topology on $X$.

Proposition 1.2: (Proposition 3.6.2 [1]) Let $X$. be a poset, we have:

(1) $X$ and $\phi$ are Scott-closed;

(2) Arbitrary intersection of Scott-closed sets is Scott-closed; and

(3) Finite union of two of Scott-closed sets is Scott-closed. Then the family of all Scott-open sets in a poset $X$ is a topology on $X$. Furthermore it is $T_{\mathrm{o}}$-topology.

Proposition 1.3: (Proposition 3.5.3(3) [1]) In a domain $(X, \leq)$. Let $F$ be a finite set $X$. Then $\downarrow F$ is d-closed subset.

\section{Definition 1.4}

For any poset $X$ consider the following topologies:

(1) $\delta_{d}=\{A \subseteq X: A$ is $d-$ open $\}$ is a topology on $X$ (see proposition 3.5.2 [1]) in the case of $X$ is a domain) and is called the directed topology) ( $d$-topology for short);

(2) $\delta_{A \mid x}=\{A \subseteq X: A$ isanuppersubset $\}$ is a topology on $X([1])$ in the case of $X$ is a domain) and is called the Alexandroff topology) (Alextopology for short);

(3) $\delta_{S}=\{A \subseteq X: A$ isScott opensubset $\}$ is a topology on $X[1-3]$ and is called the Scott- topology);

(4) The upper topology on $X$ is denoted by $\delta_{U}$ and is the topology generated by subbasis $\{X-\downarrow x: x \in X\}$ [2];

(5) TheLower topology on $X$ is denoted by $\delta_{L}$ and is the topology generated by subbasis $\{X-\uparrow x: x \in X\}$ [2];

(6) The interval topology $\delta_{I}$ on $X$ is the supremum of $\delta_{U}$ and $\delta_{L}$ i.e., $\delta_{I}=\delta_{U L}[2]$;

(7) The Lowson topology $\delta_{L S}$ on $X$ is the supremum of $\delta_{S}$ and $\delta_{L}$ i.e., ${ }_{L S}=\delta_{L} \delta_{S}[2]$.

(8) Let $(X, \delta)$ be a topological space, and let $A \subseteq X$, then the closure of $\lambda$ denoted by $c l_{\delta}()$ defined as follows $c l_{\delta}(\lambda)=\cap\{F \subseteq X: F$ is $\delta$ - closed and $\lambda \subseteq F\}[14]$.

Theorem 1.1: (Remark 1.4 (ii) [7]). Let $X$ be a complete lattice. Then, $\forall x \in X, \downarrow x . \in c l_{\delta_{S}}(\{x\})$.

Proposition 1.4: (Theorem 6.1.2 [1]). A point $x$ is an isolated iff the upper cone $\uparrow x$ is Scott-open.
Proposition 1.5: (Proposition 6.7.7 [1]). Let $(X, \leq)$ be a continuous domin. Then $\forall x \in X$, the set $\Uparrow x$ is Scott-open.

Proposition 1.6: (Proposition 6.7.8 [1]). In a continuous domain $X$, $x<<y$ holds iff there is a Scott-open set $\bigcirc$ s.t. $y \in \bigcirc \subseteq \uparrow x$.

Theorem 1.2: (Theorem 6.7.9 [1]). A domain $(X, \leq)$ is continuous iff it has the local upper cone property: forevery $x \in X$, and for every $\bigcirc \in \delta_{S}$, s.t. $x \in \bigcirc, \exists \bigcirc^{\prime}$ and $x^{\prime} \in X$ s.t., $x \in \bigcirc^{\prime} \subseteq \uparrow x^{\prime} \subseteq \bigcirc$.

\section{Definition 1.5}

A space $X$ is called a $T_{0}$-space iff for two distinct points $x, y \in X$, $\exists \lambda \subseteq X$ s.t. either $x \lambda, y \in X-\lambda$ or $y \lambda, x \in X-\lambda[14]$.

\section{Definition 1.6}

Let $\left(X, \leq_{1}\right)$ and $\left(X, \leq_{2}\right)$ be TRS and let $f:\left(X, \leq_{1}\right) \rightarrow\left(Y,,_{2}\right)$ be a function [2]. Then:

(1) $f$ is monotone iff $f(\lambda) \leq_{2} f(\mu)$, whenever $\lambda \leq_{1}$

(2) $f$ is called Scott-continuous iff $\forall G \in\left(\delta_{S}\right)_{Y}, f^{-1}(G) \in\left(\delta_{S}\right)_{X}$, where $\left(\delta_{S}\right)_{Y}\left(\operatorname{resp} .\left(\delta_{S}\right)_{X}\right)$ is the Scott-topology on $Y$ induced by $\leq_{2}$ (resp. on $X$ induced by $\leq_{1}$ ).

\section{Definition 1.7}

Let $\left(X, \leq_{1}\right)$ and $\left(X, \leq_{2}\right)$ be TRS and let $f:\left(X,\left(\delta_{A \mid x}\right)_{X}\right) \rightarrow\left(Y,\left(\delta_{A \mid x}\right)_{Y}\right)$ is called Alexandroff-continuous iff $f$ is monotone, $\left(\delta_{A \mid x}\right)_{X}$ (resp. $\left.\left(\delta_{A \mid x}\right)_{Y}\right)$ is the Alexandroff-topology on $X$ (resp., on $Y$ ) induced by $\leq_{1}\left(\right.$ resp. $\left.\leq_{2}\right)$.

\section{Directed ${ }^{*}-$ Open, Scott ${ }^{*}-$ Open Sets and Transitive Binary Relational Sets}

\section{Definition 2.1}

Atransitive binary relational set (TRS for short) is a pair $(X, \leq)$ cwhere $X \phi$ and ' $\leq$ 'is a transitive binary relation set.

Example 2.1: Partialy order set, pre-orderd set, continuous information system, equivalence set and abstract basis is TRS.

Remark 2.1: Abstract basis $\Rightarrow$ Continuous information system, the converse is not true, so give the following example

Example 2.2: $\operatorname{Let} X=\{x, y, z\}$ and let $\leq=\{(x, x),(y, y),(x, z),(y, z)\}$ because

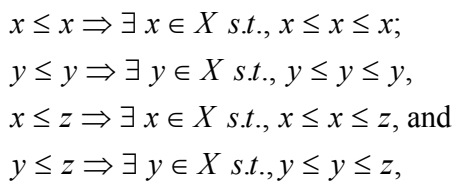

then $\leq$ transitive and interpolative. Now, Let $\lambda=\{x, y\}$. Then $x \leq z$ and $y \leq z$, but $\forall a \in X, a \nless \leq z$. Hence $(X, \leq)$ i s not abstract basis.

\section{Definition 2.2}

Let $(X, \leq)$ be a TRS. Then:

(1) $D$ is called directed subset of $X$ iff $D \neq \phi$ and for every distinct points $(x \neq y) \in D, \exists z \in D \cap u b(\{x, y\})$;

(2) $D$ is called $S$-directed subset of $X$ iff $D$ is a directed subset and $\forall x \in D,(\{x\}) \cap D \neq \phi$.

Remark 2.2: $S$-directed subset $\Rightarrow$ directed subset, the converse is not true, so give the following example. 
Example 2.3: Let $\leq$ be TRS on $X=\{x, y, z, l, m\}$ define by $x \leq y$. Then $\{x\}$ is directed subset but not $S$-directed subset.

\section{Definition 2.3}

Let $(X, \leq)$ be a TRS. Then: $D$ of $\lambda$

(1) A subset $\lambda$ of $X$ is called $d^{*}$-closed iff for every $S$-directed subset

$(D) \subseteq \lambda$ and $\forall x \lambda,(\{x\}) \subseteq \lambda$,

(2) A subset $\lambda$ of $X$ is called Scott ${ }^{*}$-closed iff $\lambda$ is $d$-closed lower subset,

(3) A subset $\lambda$ of $X$ is called $d$-open (resp. Scott-open) iff $\lambda^{c}$ is $d$ closed (resp. Scott-closed).

Theorem 2.1: Let $(X, \leq)$ be a TRS. Then:

(1) A subset $\mu$ of $X$ is called $d$-open iff for every $S$-directed subset $D$ of $X$, s.t. $(D) \cap \mu \neq \phi$,

and $\forall x \in X,(\{x\}) \cap \mu \neq \phi, x \notin \mu$, and

(2) A subset $\mu$ of $X$ is called Scott*-open iff $\mu$ is $d^{*}$-open upper subset.

Proof: (1) The proof is obtained logically as follows:

Let $E_{S}$ be the set of all $S$-directed subset of $X$.And Let $P \equiv D$ is $S$-directed subset ' $\equiv D \in E_{S}^{\prime}$;

$$
\begin{gathered}
Q \equiv^{\prime} D \subseteq \lambda^{\prime} ; \\
R \equiv(D) \subseteq \lambda^{\prime} ; \\
L \equiv^{\prime} x \in X^{\prime} ; \\
S \equiv^{\prime} x \in \lambda^{\prime} ; \\
H \equiv(\{x\}) \subseteq \lambda^{\prime} .
\end{gathered}
$$

Now, $\lambda$ is $d^{*}$-closed

$\equiv P \wedge(Q \Rightarrow R) \wedge L \wedge(S \Rightarrow H) \equiv P \wedge(\neg R \Rightarrow \neg Q) \wedge L \wedge(\neg H \Rightarrow \neg S) \equiv \lambda^{c}$

is $d^{*}$-open. Then $\lambda^{c}$ is $d^{*}$-open

$\equiv\left(D \in E_{S}\right) \wedge\left(\left((D) \cap \lambda^{c}\right) \neq \phi \Rightarrow\left(D \cap \lambda^{c}\right) \neq \phi\right) \wedge(x \in X) \wedge\left((\{x\}) \cap \lambda^{c}\right) \neq \phi \Rightarrow x \notin \lambda^{c}$.

Then $\mu$ is $d$-open iff $\forall D \in E_{S}$ s.t. $(D) \cap \mu \neq \phi ; \quad$ and $\forall x \in X,(\{x\}) \cap \mu \neq \phi, x \notin \mu$.

(2) By the same fashion

Theorem 2.2: Let $(X, \leq)$ be a TRS. If $\lambda$ is $d$-closed (resp. $d$-open) subset of $X$ then $\lambda$ is $d^{*}$-closed (resp. $d^{*}$-open).

Proof: Only prove the stament. Let $D$ be an $S$-directed subset of $\lambda$. So since $\lambda$ is $d$-closed, then $(D) \subseteq \lambda$. Also, $\forall x \in \lambda\{x\}$, directed subset of $\lambda$ so that $(\{\mathrm{x}\} \subseteq \lambda$. Hence $\lambda$ is $d$-closed.

Corollarly 2.1: From Theorem 2.1. Let $(X, \leq)$ be a TRS. If $\lambda$ is Scottclosed (resp. Scott-open) subset of $X$, then $\lambda$ is Scott*-closed (resp. Scott ${ }^{*}$-open).

Open problem if $\lambda$ is $d^{*}$-open subset of a TRS, then $\lambda$ is $d$-open.

Theorem 2.3: Let $(X, \leq)$ be a pre-orderd set and $\lambda \subseteq X$. Then:

(1) $\lambda$ of $X$ is directed subset iff $\lambda$ is an $S$-directed subset of $X$;

(2) $\lambda$ is $d$-open (resp. $d$-closed, Scott-closed, Scott-open) subset of $X$ iff $\lambda$ is $d^{*}$-open (rep. $d^{*}$-closed, Scott ${ }^{*}$-closed, Scott ${ }^{*}$-open).

Proposition 2.1: Let $\lambda, \mu \in X$. If $\mu$ directed subset and cofinal in $\lambda$, then $\lambda$ is directed subset and $(\lambda)=()$.
Proof: First, we prove that $\lambda$ is direct subset. Since $\subseteq \lambda$, then $\lambda \neq \phi$. Let $l, m \in \lambda$ s.t. $\quad l \neq m$. Then $\exists b_{1}, b_{2} \in \mu$ s.t. $l \leq b_{1}, m \leq b_{2}$ and $\exists b \in \lambda \cap u b(\{l, m\})$. Hence $\lambda$ is directed subset. Scound, one can deduce that $u b(\mu) \subseteq u b(\lambda)$. Conversely, $y \notin u b(\lambda) \Rightarrow \exists a \in \lambda$ s.t. $a \not \leq y \Rightarrow \exists a \in \downarrow(\mu) \Rightarrow \exists b \in \mu$ s.t. $a \leq b$ and $a \not \leq y \Rightarrow \exists b \in \mu$ s.t. $b \not \leq y \Rightarrow y \notin u b(\mu)$. Hence $u b(\mu) \subseteq u b(\lambda)$. Thus $(\lambda)=()$.

The following theorem is a generalization of the corresponding result in Proposition 1.1 (Proposition 3.5.2 [1]).

Theorem 2.4: Let $(X, \leq)$ be a TRS. If $\forall x \in X,(\{x\}) \neq \phi$, then $\delta_{d^{*}}=\left\{\lambda \subseteq X: \lambda\right.$ is $d^{*}$-open $\}$ is a topology on $X$ (called directed ${ }^{*}$ topology on $X)$.

Proof: (1) Clearly $X$ and $\phi$ are $d^{*}$-closed sets. So $X$ and $\phi$ are $d^{*}$-open sets;

(2) Let $\lambda$ and $\mu$ be $d^{*}$-open sets. Then $\lambda^{c}$ and $\mu^{c}$ be $X$ be $d^{*}$-closed sets. Let $D$ be an $S$-directed subset of $\lambda^{c} \cup \mu^{c}$. Thus $D=\left(D \cap \lambda^{c}\right)$ or $\left(D \mu^{c}\right)$. We need to prove that either $\left(D \cap \lambda^{c}\right)$ or $\left(D \mu^{c}\right)$ is cofinal in $D$. Suppose $D \lambda^{c}$ is not cofinal in $D$, then $\exists d_{\circ} \in D$ s.t. $\forall a \in D \cap \lambda^{c}, d_{\circ} \nless \leq a$. If $d_{0} \in \lambda^{c}$, then $\left(\left\{d_{0}\right\}\right) \subseteq \lambda^{c}$. Thus $\exists m \in D \cap \lambda^{c}, d_{\circ} \leq \leq a$ s.t. $d_{\circ} \leq m$. Acontraduction. Hence $d_{\circ} \in \mu^{c}$. Let $d \in D$ s.t.d $\neq d_{\circ}$. Then $\exists d^{\prime} \in D \cap u b\left(\left\{d_{\circ}, d\right\}\right)$. If $d \lambda^{c}$, then $d_{\circ} \leq d^{\prime}$ which leads to a contaduction. So, $d^{\prime} \in \mu^{c}$, i.e., $D \subseteq \downarrow D \cap \mu^{c}$. Hence $D \mu^{c}$ is cofinal in $D$. Now we prove that $D \mu^{c}$ is directed. Let $b_{1}, b_{2} \in D \cap \mu^{c}$ s.t. $b_{1} \neq b_{2}$. Thus $\exists d \in D \cap u b\left(\left\{b_{1}, b_{2}\right\}\right)$. Thus $\exists k \in D \cap \mu^{c}$ s.t. $d \leq k$. So, $k \in\left(D \cap \mu^{c}\right) \cap u b\left(\left\{b_{1}, b_{2}\right\}\right)$. Hence $D \cap \mu^{c}$ is directed. From Proposition 2.1. $(\mathrm{D})=\left(D \cap \mu^{c}\right)$. Now $D \cap \mu^{c}$ is $S$-directed (Indeed, let $l \in D \cap \mu^{c}$. Since $D$ is $S$-directed, $\exists m \in(\{l\})$ s.t. $m \in D$. Also, since $\mu^{c}$ is $d$-closed, then $(\{l\}) \subseteq \mu^{c}$. Thus $m \in D \cap \mu^{c}$, i.e., $\left(D \cap \mu^{c}\right) \cap(\{l\}) \neq \phi$. Hence $D \mu^{c}$ is directed). Then $(D)=\left(D \cap \mu^{c}\right) \subseteq \mu^{c} \subseteq \mu^{c} \cap \lambda^{c}$. Also, if $x \in \mu^{c} \lambda^{c}$, then $(\{x\}) \subseteq \mu^{c} \lambda$. So $\mu^{c} \cup \lambda^{c}$ is $d^{*}$-closed so that $\lambda \cap \mu$ is $d$-open, and (3) Let $\left\{\lambda_{i}: i \in I\right\}$ be a family of $d^{*}$-open subsets of $X$. Then $\left\{\lambda_{i}^{c}: i \in I\right\}$ is a family of $d$-closed subsets of $X$. Let $D$ be an $S$-directed subset of ${ }_{i \in I} \lambda_{i}^{c}$. So, $D \subseteq \lambda_{i}^{c} \forall i \in I$. Then $(D)=\lambda_{i}^{c} \quad \forall i \in I$. So that $(D)={ }_{i \in I} \lambda_{i}^{c}$. Let $l \in \in_{i \in I} \lambda_{i}^{c}$, then $(\{l\})=\lambda_{i}^{c} \forall i \in I$. Thus $(\{l\})=\lambda_{i}^{c} \forall i \in I$. So that $(\{l\})=_{i \in I} \lambda_{i}^{c}$. Then ${ }_{i \in I} \lambda_{i}^{c}$ is $d$-closed. Hence ${ }_{i \in I} \lambda_{i}$ is $d$-open.

Proposition 2.2: (1) $X$ and $\phi$ areLower (resp. upper) subsets; and

(2) If $\left\{\lambda_{i}: i \in I\right\}$ be a family of Lower (resp. upper) subsets of $X$, then ${ }_{i \in I} \lambda_{i}$ and ${ }_{i \in I} \lambda_{i}$ are Lower (resp. upper) subsets.

Theorem 2.5: Let $(X, \leq)$ be a TRS. If $\forall x \in X,(\{x\}) \neq \phi$, then

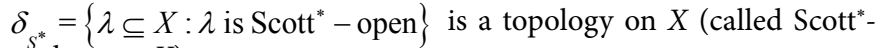
topology on $X)$.

Proof: Follow from Proposition 2.2. and Theorem 2.4.

Remark 2.2: Each pre-orderd set is a TRS satisfies $\forall x X,(\{x\}) \neq \phi$. the converse is not true, so give the following example.

Example 2.4: Let $\leq$ be a binary relation on $X=\{x, y, z\}$ define by $x \leq z$, $y \leq z$ and $z \leq z$. Then $(X, \leq)$ is TRS and $\forall x \in X,(\{x\})=\{z\} \neq \phi$. But $(X, \leq)$ is not pre-orderd set because $\leq$ is not reflexive.

Theorem 2.6: Let $(X, \leq)$ be a TRS and let $x \in X$. Then $\downarrow x$ is Scott-closed.

Proof: (1) Let $y \downarrow(\downarrow x)$. then $\exists z \downarrow x$ s.t. $y \leq z$ so that $y \in \downarrow x$. Hence $\downarrow x$ is a lower subset of $X$. 
Citation: Khalaf MM (2017) Directed*-topology and Scott*-topology on Transitive Binary Relational Sets. J Generalized Lie Theory Appl 11: 284 . doi: 10.4172/1736-4337.1000284

Page 4 of 6

(2) Let $D$ be a directed subsets of $\downarrow x$ and let $m \in(D)$. Now $x \in u b(D)$ and thus $m \leq x$ i.e., $m \in \downarrow x$. Then $(D) \subseteq \downarrow x$. Hence $\downarrow x$ is d-closed. From (1) and (2) we have $\downarrow x$ is Scott-closed.

Corollarly 2.2: Let $(X, \leq)$ be a TRS s.t. $\forall x \in X,(\{x\}) \neq \phi$. Then $\forall x \in X, X-\downarrow x \in \delta_{S}$.

Theorem 2.7: Let $(X, \leq)$ be pre-orderd set. Then $\downarrow x=c l_{\delta_{S}}(\{x\})$.

Proof: From Corollarly 2.2. $X-\downarrow x \in \delta_{s}$. Since $x \leq x, x \leq x$,

then $c l_{\delta_{S}}(\{x\})=\left\{F \subseteq X: F\right.$ is $\delta_{S}-$ closed and $\left.\{x\} \subseteq F\right\} \subseteq \downarrow x \subseteq\left\{\downarrow F \subseteq X: F\right.$ is $\delta_{s}-\operatorname{closed}$ and $\left.\{x\} \subseteq F\right\}$

$=\left\{F \subseteq X: F\right.$ is $\delta_{S}-$ closed and $\left.\{x\} \subseteq F\right\}=c l_{\delta_{S}}(\{x\})$.

The following theorem is a generalization of the corresponding result in Proposition 1.3 (Proposition 3.5.3(3) [1]).

Theorem 2.8: Let $F$ be a finite set in pre-orderd set $(X, \leq)$. Then $X-\downarrow F \in \delta_{S}$.

Proof: Since $\quad z \in \downarrow F \Leftrightarrow \exists x \in F$ s.t. $z \leq x \Leftrightarrow z \in\{\downarrow x: x \in F\}$. Then On can deduce $\downarrow F=\{\downarrow x: x \in F\}$. From Theorem 2.7. $\forall x \in X, \downarrow x$ is Scott-closed. Since the union of finitaly number of $d$-closed is Scott-closed subset, then $\downarrow F$ is Scott-closed subset so that $X-\downarrow F \in \delta_{S}$.

\section{Definition 2.4}

Let $(X, \leq)$ be a TRS. and let $(X, \delta)$ be a topological space, Then $(X, \leq, \delta)$ is called a topological TRS.

\section{Definition 2.5}

Let $(X, \leq, \delta)$ is called a topological TRS. The topological space $(X, \delta)$ is called transitive- $T_{\circ}(T R S-T$ for short) iff $\forall x, y \in X$, s.t. either $x \not \leq y$ or $y \not \leq x, \exists u \in \delta$ s.t. $x \in u, y \notin u$ or $y \in u, x \notin u$.

Lemma 2.1: Let $(X, \leq)$ be a TRS. and let $\delta$ be a topology on $X$, Then $(X, \delta)$ is $T R S-T_{\mathrm{o}}$ iff $\forall x, y \in X$, s.t. $x \not \leq y$ or $y \leq x$, either $x \in c l_{\delta}(\{y\})$ or $y \in \operatorname{cl}_{\delta}(\{x\})$. Then:

Proposition 2.3: Let $(X, \leq)$ be a TRS. and let $\delta$ be a topology on $X$,

(1) If $\leq$ is antisymmetric and $(X, \delta)$ is $T R S-T_{0}$ then $(X, \delta)$ is $T$

(2) If $\leq$ is reflexive and $(X$,$) is T_{\text {。 }}$ then $(X, \delta)$ is $T R S-T$.

The following theorem is a generalization of the corresponding result in Theorem 1.1 (Remark 1.4 (ii) [7]).

Theorem 2.9: Let $(X, \leq)$ be pre-orderd set, then $\left(X, \delta_{S}\right)$ is $T R S-T_{\text {。 }}$.

Proof: $\forall x, y \in X$, s.t. either $x \not \leq y$ or $y \not \leq x$, Then $x \downarrow y$ or $y \downarrow x$. From Theorem 2.7, $x \in c l_{\delta_{S}}(\{y\})$ or $y \in c l_{\delta_{S}}(\{x\})$. Hence from Lemma 2.1, $\left(X, \delta_{S}\right)$ is $T R S-T$.

Theorem 2.10: Let $(X, \leq)$ be pre-orderd set. Then a specialization pre-ordered relation $\lesssim_{\delta_{S}}$ induced by $\delta_{S} \leq$.

Proof: $x \lesssim_{\delta_{S}} y \Leftrightarrow x \in \operatorname{cl}_{\delta_{S}}(\{y\})=\downarrow y \Leftrightarrow x \leq y$.

\section{Interactions between a Continuous TRS and Its Scott*- Topology}

The following theorem is a generalization of the corresponding result in Proposition 1.4 (Theorem 6.1.2 [1]).

Theorem 3.1: (1) Let $(X$,$) be a TRS and x X$ is an isolated point, then $\uparrow x$ is Scott-open,

(2) Let $(X, \leq)$ be a $T R S$ and for some $x \in X, \uparrow x$ is d-open subset of $X$, then $x$ is an isolated point.

Proof: (1) Let $D$ be a directed subset of $X$ and assume that $(D) \cap \uparrow x \neq \phi$. Thus $\exists y \in X$ s.t. $y \in \uparrow x$ and $y(D)$. Then $x \in \downarrow(D)$. Since $x<<x$, then $\exists d \in D$ s.t. $d \in \uparrow x$. Hence $D \cap \uparrow x \neq \phi$. Then $\uparrow x$ is d-open. Since $\uparrow x$ is upper subset, then $\uparrow x$ is Scott-open.

(2) Let $D$ be a directed subset of $X$ and $x \downarrow(D)$. Then $\exists m \in(D)$ s.t. $x \leq m$ so that $m \in(D) \cap \uparrow x$. Since $\uparrow x$ is d-open, then $D \cap \uparrow x \neq \phi$, i.e., $\exists d \in D$ s.t. $x \leq d$.

Hence $x<<x$.

Corollarly 3.1: (1) Let $(X$,$) be a TRS s.t. \forall x \in X,(\{x\}) \neq \phi$. if $x \in X$ is an isolated point, then $\uparrow x \in \delta_{S}$; and

(2) $\operatorname{If}(X, \leq)$ is a pre-orderd set and for some $x \in X$, $\uparrow x \delta_{S}$ (moreover $_{d}$ ) then is an isolated point.

Proposition 3.1: Let $(X, \leq)$ be a TRS. Let $x, y, z \in X$. Then:

(1) If $x \leq y$ and $y<<z$, then $x<<z$;

(2) If $x<<y$ and $y \leq z$, then $x<<z$;

(3) If $(\{y\}) \phi$ and $x<<y$, then $x y$;

(4) $\operatorname{If}(\{y\}) \phi$ or $(\{z\}) \neq \phi, x<<y$ and $y<<z$ then $x<<z$.

Proof: (1) Let $D$ be a directed subset of $X$ s.t. $z \downarrow(D)$. Then $\exists d D$ s.t.yd. Then $x d$ and hence $x<<z$.

(2) Let $D$ be a directed subset of $X$ s.t. $z \downarrow(D)$. Then $\exists k \in(D)$ s.t. $z \leq k$. Thus $y k$ and so $y \in \downarrow(D)$. Therefore $\exists l \in D$ s.t. $x \leq l$. hence $x<<z$.

(3) Let $\mathrm{D}=\{y\}$ and assume that $x<<y$. Then $\exists d \in D$ s.t. $x \leq d$ but $y=d$ Thus $x \leq y$.

(4) The proof follow directly from (1) and (3) above.

The following theorem is a generalization of the corresponding result in Proposition 1.5 (Proposition 6.7.7 [1]).

Theorem 3.2: Let $(X, \leq)$ be a TRS. If ' $<<$ ' is interpolative, then $\forall x \in X, \Uparrow x$ is Scott-open.

Proof: First let $z \uparrow(\Uparrow x)$. Then $\exists y x$ s.t. $y z$. From Proposition 3.1.(2) $z \in \Uparrow x$. Hence $\Uparrow x$ is upper subset of $X$. Second Let, $D$ be a directed subset of $X$ with $\Uparrow x \cap \downarrow(D) \neq \phi$. Then $\exists z \leq X$ s.t. $z \in \downarrow(D)$ (because for each element $z \in(D), z \leq z)$ and $z \in \Uparrow x$. So, $\exists y \in X$ s.t. $x \ll y \ll z$ and so that $\exists d \in D$ s.t. $y \leq d$. From Proposition 3.1.(2) $d \Uparrow x$. Hence $D \cap \Uparrow x \neq \phi$.

\section{Definition 3.1}

$(X, \leq)$ is a continuous TRS iff $\forall x X$, the following conditions are satisfied:

(1) $(\{x\}) \neq \phi$

(2) $\Downarrow_{x}$ be a directed subset of $X$, and

(3) $x \in \downarrow\left(\left(\left\{\left(\Downarrow_{a}\right): a \in \Downarrow_{x\}}\right)\right)\right.$.

Corollarly 3.2: If $(X, \leq)$ is a continuous $T R S$, then $\forall x \in X, \Uparrow x \delta_{S}$.

Theorem 3.3: Let $(X, \leq)$ be a pre-orderd set and let $x, y X$. If $\exists \bigcirc$ (open set) in $\delta_{s}$ s.t. $y \in O \subseteq \uparrow x$, then $x<<y$.

Proof: Let $D$ be a directed subset of $X$ s.t $y \in \downarrow(D)$. Then $\exists m(D)$ 
Citation: Khalaf MM (2017) Directed*-topology and Scott*-topology on Transitive Binary Relational Sets. J Generalized Lie Theory Appl 11: 284 . doi: 10.4172/1736-4337.1000284

Page 5 of 6

s.t. $y \leq m$. Since $y \in \uparrow y \uparrow \bigcirc \subseteq \bigcirc$, then $m \in(D) \cap \bigcirc$.i.e. $(D) \cap \bigcirc \neq \phi$. Then $\exists d \in D$ s.t. $d \in \bigcirc \subseteq \uparrow x$, i.e. $x \leq d$. Hence $x<<y$.

The following theorem is a generalization of the corresponding result in Proposition 1.6 (Proposition 6.7.8 [1]).

Theorem 3.4: Let $(X, \leq)$ be a TRS. s.t. $\forall z X,(\{z\}) \neq \phi$ and assume that ' $<<$ ' is interpolative. If $\forall x, y \in X$, s.t. $x \ll y$, then $\exists$ a Scott-open subset $\bigcirc$ of $X$ s.t. $y \in \bigcirc \subseteq \uparrow x$.

Proof: From Theorem 3.2. $\Uparrow x$ is Scott-open. Let $y \in \Uparrow x$ i.e. $x<<y$. From Proposition 3.1.(3), $y \in \Uparrow x$. Hence $y \in \Uparrow x \uparrow x$.

Theorem 3.5: Let $(X, \leq)$ be a continuous pre-orderd set. Then $\forall x \in X$, and $\forall \bigcirc \in \delta_{S}$ with $z \in \bigcirc$ for some $z \in(\Downarrow x), z \in \bigcirc^{\prime} \in \delta_{S}$ and $x^{\prime} \in X \quad$ s.t. $y \in \bigcirc^{\prime} \subseteq \uparrow x^{\prime} \subseteq \bigcirc$.

Proof: Let $\forall x X$ and let $\bigcirc \in \delta_{s}$ with $z \in$ Ofor some $z(\Downarrow x)$. Then $(\Downarrow x) \cap \bigcirc \neq \phi$. Thus $\exists x^{\prime} \in \Downarrow x \cap \bigcirc$ so that $\uparrow x^{\prime} \subseteq \uparrow \bigcirc \subseteq \bigcirc$. From Theorem 3.4. and since $x^{\prime} \subseteq x, \exists \bigcirc^{\prime} \in \delta_{S} \quad$ s.t. $x \in \bigcirc^{\prime} \subseteq \uparrow x^{\prime} \subseteq \uparrow \bigcirc \subseteq \bigcirc$.

Theorem 3.6: Let $(X, \leq)$ be domain pre-orderd set. If $\forall x \in X$, and $\forall \bigcirc \in \delta_{s}, \exists \bigcirc^{\prime} \in \delta_{s}$ and $\exists x^{\prime} \in X$ s.t. $x \in \bigcirc^{\prime} \subseteq \uparrow x^{\prime} \subseteq \bigcirc$, then $\exists$ a directed subsets $D$ of $\Downarrow x$ s.t. $x \in \downarrow(D)$.

Proof: Let $D=\left\{u \in X: \exists \bigcirc_{u} \in \delta_{S}\right.$ with $\left.\quad x \in \bigcirc_{u} \subseteq \uparrow u\right\}$. From Theorem 3.3, $D \subseteq \downarrow_{x}$. Since $X$ iteslf is Scott-open and $x X$, then there are $y \in X$ and $\bigcirc_{y} \in \delta_{s}$ s.t. $x \in \bigcirc_{y} \subseteq \uparrow y$. Then $D \phi$. Let $u, v \in D$ s.t.uv. Then, there $\exists \bigcirc_{u}, \bigcirc_{v} \in \delta_{S}$ s.t. $x \in \bigcirc_{u} \subseteq \uparrow u$ and $x \in \bigcirc_{v} \subseteq \uparrow v$. Since $x \in \bigcirc_{u} \cap \bigcirc_{v}$, there $\exists w X$ and $\bigcirc_{w} \delta_{S}$ s.t. $x \in \bigcirc_{w} \subseteq \uparrow w \subseteq \bigcirc_{u} \cap \bigcirc_{v}$. Then $w D$ and $w \uparrow u \cap \uparrow v$. So $u \leq w$ and $v \leq w$. Then $D$ is directed subsets of $\Downarrow_{x}$. Now there are $y \in X$ and $\bigcirc_{y} \in \delta_{S}$ s.t. $x \in \bigcirc_{y} \subseteq \uparrow y \subseteq \bigcirc$. Thus $y D \cap \bigcirc$. Let $m \in(D)$, then $y m$. Since $\bigcirc$ is upper subset, then $m \bigcirc$. Now $x \lesssim_{\delta_{S}} m$. From Theorem 2.11, $x m$. Hence $x \downarrow(D)$.

The following theorem is a generalization of the corresponding result in Theorem 1.2 (Theorem 6.7.9 [1])

Theorem 3.7: Let $(X, \leq)$ be domain pre-orderd set. Assume that If $\forall x X$, and $\forall \bigcirc \in \delta_{S}, \exists z \in(\Downarrow x) \cap \bigcirc$. Then the following statments are equivalent:

(1) $(X, \leq)$ be a continuous TRS, and

(2) (a) ' $<<$ ' is interpolative,

(b) $\forall x X, \forall \bigcirc \in \delta_{s}$ with $x \in O$ there are $\bigcirc^{\prime} \in \delta_{s}$ and $x \in X$ s.t. $x \in \mathrm{O}^{\prime} \subseteq \uparrow x^{\prime} \subseteq \mathrm{O}$.

Proof: Applying Theorem 3.4, Theorem 3.5, Theorem 3.6. the results holds.

\section{An Application on Topological Spaces}

From Theorem 2.6, one can assign for any topology $\delta$, a new topology $S(\delta)$ where $S(\delta)$ is Scott-topology induced by the specialization pre-ordered relation $\lesssim_{\delta}$ induced by $\delta$.

Theorem 4.1: Let $(X, \delta)$ be a topological space Then:

(1) $(X, S(\delta))$ be a topological space is TRS-T.w.r.t, the pre-ordered relation $\lesssim_{\delta}$; and

(2) If $(X, \delta)$ is $T_{\text {o }}$-space, then $(X, S(\delta))$ so is.

Proof: (1) The result is a corollary from Theorem 2.9.

(2) From Proposition 4.3.3 [1], if $(X, \delta)$ is $T_{0}$-space, then $\leq \delta$ is a partially ordered relation. So, from Theorem 2.9. and proposition 2.3 . one can have the $(X, S(\delta))$ is $T_{\mathrm{o}}$-space.

Theorem 4.2: Let $(X, \leq)$ be a pre-orderd set. Then $S\left({ }_{S}\right)={ }_{S}$.

Proof: The result follows from Theorem 2.10.

\section{More Topologies on TRS and More on Functions between TRS}

Proposition 5.1: (page 10 [2]). In (X,), let $d \in X$. Then:

(1) $\downarrow d$ is a principle ideal; and

(2) $\uparrow d$ is a principle filter.

Definition 5.1

Let $(X, \leq)$ be a TRS. $\forall \in X,(\{x\}) \neq \phi$. Define topologies:

(1) $\delta_{\lambda \mid x}=\{\lambda \subseteq X: \lambda$ is anuppersubset $\}$ (see Proposition 5.1.) is a topology on $X$ called the Alexandroff topology;

(2) The upper topology on $X$ is induced by $\delta_{\alpha}$ and is the topology generated by subbasis $\{X-\downarrow x: x \in X\}$;

(3) The lower topology on $X$ is denoted by $\delta_{\beta}$ and is the topology generated by subbasis $\{X-\uparrow x: x \in X\}$;

(4) The interval topology $\delta_{\chi}$ on $X$ is the supremum of $\delta_{\alpha}$ and $\delta_{\beta}$, i.e $\delta_{\chi}=\delta_{\alpha \beta}$

(5) The Lowson topology $\delta_{\beta S}$ on $X$ is the supremum of $\delta_{\beta}$ and $\delta_{S}$, i.e $\delta_{\beta S}={ }_{\beta} \delta_{S}$

Definition 5.2

Let $\left(X, \leq_{1}\right)$ and $\left(X, \leq_{2}\right)$ be TRS and let $\lambda, \mu \in X$ and let $f:\left(X, \leq_{1}\right) \rightarrow\left(Y,{ }_{2}\right)$ be a function. Then $f$ is monotone iff $f(\lambda) \leq_{2} f(\mu)$, whenever $\lambda \leq_{1}$, and $\mu \leq_{1}$

Lemma 5.1: If $\left(X, \leq_{1}\right)$ and $\left(X, \leq_{2}\right)$ be are pre-order sets then the function $f:\left(X, \leq_{1}\right) \rightarrow\left(Y,,_{2}\right)$ is TRS monotone iff it is monotone.

Proof: Obvious

Theorem 5.1: Let $(X, \leq)$ be pre-orderd set. Then the following statments are hold:
(1) $\delta_{\alpha} \leq \delta_{S}$
(2) $\delta_{S} \leq \delta_{\lambda \mid x}$
(3) $\delta_{S} \leq \delta_{d}$
(4) $\delta_{S} \leq \delta_{\beta S}$
(5) $\delta_{\beta} \leq \delta_{\beta S}$
(6) $\delta_{\alpha} \leq \delta_{\chi}$
(7) $\delta_{\beta} \leq \delta_{\chi}$
(8) $\delta_{S}=\delta_{d} \cap \delta_{\lambda \mid x}$
(9) $\delta_{\alpha} \leq \delta_{\lambda \mid x}$
(10) $\delta_{\alpha} \leq \delta_{d}$
(11) $\delta_{\alpha} \leq \delta_{\beta S}$.

In the following Theorem we give a characterization of Alexandroffcontinuous between TRS.

Theorem 5.2: Let $\left(X, \leq_{1}\right)$ and $\left(X, \leq_{2}\right)$ be TRS and let $f:\left(X, \leq_{1}\right) \rightarrow\left(X,_{2}\right)$ be a function. Then $f$ is Alexandroff-continuous iff $f$ is monotone.

Proof: $(\Rightarrow)$ Let $\lambda, \mu \in X$ s.t. $\lambda \leq$. Since $\downarrow f(\mu)$ is a lower set in $Y$, then $f^{1}(\downarrow f(\mu))$ is a lower set in $X$. From the fact $\mu \in f^{1}(\downarrow f(\mu))$ one can have that $\lambda \in f^{1}(\downarrow f(\mu))$. Then $f(\lambda) \leq_{2} f(\mu)$.

$(\Leftarrow)$ Let $F$ be a lower set in $Y$, i.e., an Alexandroff-closed in $Y$. We need to prove that $f^{1}(F)$ is a lower set in $X$. Let $\lambda \in \downarrow f^{-1}(F)$. Then $\exists \mu \in f^{1}(F)$ s.t., $\lambda \leq_{1}$. So, $f() \leq_{2} f(\mu)$. Since $F$ be a lower set, then $f(\lambda) \in F$, i.e., $\lambda \in f^{1}(F)$. Hence $f^{-1}(F)$ is Alexandroff-closed subset in $X$.

Lemma 5.1: Let $\left(X, \leq_{1}\right)$ and $\left(Y, \leq_{2}\right)$ be TRS and let $f:\left(X, \leq_{1}\right) \rightarrow\left(X,,_{2}\right)$ be a function and let 
Citation: Khalaf MM (2017) Directed*-topology and Scott*-topology on Transitive Binary Relational Sets. J Generalized Lie Theory Appl 11: 284 . doi: 10.4172/1736-4337.1000284

Page 6 of 6

(1) If $f$ is Scott*-continuous, then $f$ is TRS-monotone, and

(2) If for any direcrted subset $D$ of $X$ s.t. $\sup _{X}(D) \neq \phi, f\left({ }_{X}(D)\right)=_{Y}(f(D))$, then $f$ is TRS-monotone.

Proof: (1) Let $\lambda, \mu \in X$ s.t. $\lambda \leq_{1} \mu$. and $\mu \leq_{1}$. From Theorem 2.6. $\downarrow f(\mu)$ is $\operatorname{Scott}^{*}$-closed so that $f^{-1}(\downarrow f(\mu))$ is a lower set in $X$. It is dear that $\mu \in$ $f^{-1}(\downarrow f(\mu))$. Then $\lambda \in f^{1}(\downarrow f(\mu))$ so that $f(\lambda) \leq f(\mu)$.

(2) Let $\lambda, \mu \in X$ s.t. $\lambda \leq_{1}$ and $\mu \leq_{1}$. Then $D=\{, \mu\}$ is direcrted subset of $X$ and $\mu \in_{X}(D)$. So $f(\mu) \in f(X(D))=X(f(D))$. Thus $f(\mu) \geq_{2} f(\lambda)$. Hence $f$ is TRS-monotone.

From Lemma 5.1 one can have the following Corollary.

Corollary 5.1: Let $\left(X, \leq_{1}\right)$ and $\left(X, \leq_{2}\right)$ be are pre-order sets and let $f:\left(X, \leq_{1}\right) \rightarrow\left(Y, \leq_{2}\right)$ be the function. Then:

(1) If $f$ is Scott*-continuous, then $f$ is monotone, and

(2) Iffor any direcrted subset $D$ of $X$ s.t. $\mu \in_{X} \sup _{X}(D) \neq \phi, f\left({ }_{X}(D)\right)={ }_{Y}(f(D))$, then $f$ is monotone.

Lemma 5.2: Let $\left(X, \leq_{1}\right)$ and $\left(X, \leq_{2}\right)$ be TRS and let $f:\left(X, \leq_{1}\right) \rightarrow\left(Y,,_{2}\right)$ be a monotone function. If $D$ is directed subset of $X$, then $f(D)$ is a directed subset of $Y$

Proof: Let $\lambda_{1}, \lambda_{2} \in f(D)$ s.t. $\lambda_{1} \neq \lambda_{2}$ then $\exists \mu_{1}, \mu_{2} \in D$. s.t. $\mu_{1} \neq \mu_{2}$, and $f\left(b_{1}\right)=a_{1}$ and $f\left(\mu_{1}\right)=\lambda_{1}$. Since $D$ is directed, then $c \in D$. s.t. $c \geq_{1} \mu_{1}$, and $c \geq_{12}$. So, $f(c) \in f(D)$ s.t. $f(c) \geq_{2} \in f\left(\mu_{1}\right)=\lambda_{1}$ and $f(c) \geq_{2} \quad f\left(\mu_{2}\right)=\lambda_{2}$. Then $f(D)$ is a directed subset of $Y$.

\section{References}

1. Heckmann R (1999) Power domain constuctions. University Saarlandes.

2. Kummetz R (2000) Partially ordered sets with projections and their topology. Dresden University of Technology.
3. Nino-Salcedo J (1981) On continuous posets and their applications. Tulane University.

4. Abramsky S, Jung A (1994) Domain theory in the Handbook for logic in Computer Science. Clarendon Press, Oxford.

5. Branachewski B, Hofmann RE (1981) Continuous lattices. Springer-Verlag, Berlin, Heidelberg, New York.

6. Hofmann RE, Continuous Poset comletely distributive complete lattices and Hausdorff compactifications 3: 159-208

7. Hofmann RE (2006) Projective sober spaces. Continuous Lattices 3: 125-158.

8. Hofmann RE (1979) Sobrification of partially ordered sets. Semi-group Forum 17: $123-138$.

9. Lawson JD (1979) The duality of continuous posets, Houstan. J Math 5: 357386.

10. Lawson JD (1987) The Versatile continuous order. Springer-Veralg, Berlin heidelberg, New York, pp: 134-160.

11. Markowsky G (2006) A motivation and generalization of Scott's notion of a continuous lattice. Continuous Lattice 3: 298-307.

12. Eme M (2006) Scott convergence and Scott Topologies on Partially ordered sets II. 3: 61-96.

13. Zhang H (1993) Dualities of domains. Department of Mathematices, Tulane University.

14. Lipschutz S (1965) Schaum's Theory and problems of general topology Schaum series, McJRAW-HILL INT.

15. Vickers S (1993) Information systems for continuous posets, Theoretical Computer Science 114: 201-299.

16. Larsen K, Winskel G (1984) Using information systems to solve recursive domain equations effectively. Semantics of Data Types, pp: 109-129.

17. Waszhiewicz P (2002) Quantitative continuous domain. Applied Categorical Structures 11: 41-67. 